\title{
WiseNET: an ultra low-power concept for Wireless Sensor Networks
}

\author{
J.-D. Decotignie, C. Enz, V.Peiris, M.Hübner \\ Swiss Center for Electronics and Microtechnology (CSEM)
}

Jaquet-Doz 1, 2002 Neuchâtel, Switzerland, www.csem.ch

\begin{abstract}
The WiseNET system includes an ultra low-power system-on-chip (SoC) hardware platform, WiseMAC, a low power medium access control protocol (MAC) dedicated to duty-cycled radios and a cluster-tree routing protocol. These elements have been designed to meet the specific requirements of wireless sensor networks and are particularly well suited to ad-hoc and hybrid networks. The WiseNET radio offers dual-band operation (434-MHz and 868-MHz) and runs from a single 1.5-V battery. It consumes only 2.5$m W$ in receive mode with a sensitivity smaller than $-108-d B m$ at a BER of $10^{-3}$ and for a $100-\mathrm{kb} / \mathrm{s}$ data rate. In addition to this low-power radio, the WiseNET system-on-chip (SoC) also includes all the functions required for data acquisition, processing and storage of the information provided by the sensors. Ultra-low power consumption with the WiseNET system is achieved thanks to the combination of the low power consumption of the transceiver and the high energy efficiency of the WiseMAC protocol. The WiseNET solution consumes more than 250 times less power than comparable solutions based on the IEEE 802.15.4 standard.
\end{abstract}

\section{Introduction}

A wireless sensor network (WSN) consists of a number of energy-autonomous micro sensors distributed in an area of interest. Each node monitors its local environment, locally processes and stores the collected data so it can be used by other nodes. It shares this information with the other neighboring nodes by using a wireless link. Specific features of interest to the end-user can be extracted from the different information collected by several nodes by using data fusion while this information is propagated to some collection points (sinks).

The WiseNET system is the result of CSEM project initiated in 2001 to design and built a WSN solution with the lowest posssible energy consumption. This includes a low power system on chip (SoC) and a full protocol stack. The SoC combines a RF transceiver, a processor, some sensor interface and power mangement cicuitry. The protocol stack is made of Low Power Listening MAC (Medium Access Control), a cluster-tree routing protocol, a management protocol, a default application layer and a protocol to update and upgrade the node software.

Taking into account the special context of WSN, a few basic choices have been made. First, a reasonably high emission power (actually the maximum allowed in the selected bands) was chosen instead of trying to optimize the emission efficiency. This choice is justified by the fact that nodes will emit rarely and hence emission efficiency is not a high priority parameter. Second, the effort was focused in reducing the energy consumption in receive mode as well as the wake-up and turn-around times. Since a form of preamble scanning is used at the MAC layer, waking up and switching quickly has a clear impact on power consumption.

For the same reasons, the RSSI information has been carefully elaborated. As a third level of optimization, robust bit synchronization and packet filtering based on a programmable pattern has been added. Both reduce frame losses while the former additionally reduces overhearing.

This paper will describe the different parts of the system. Its performances will then be assessed from the theoretical viewpoint. The last part will give an overview of some of the deployments of this system in different applications.

\section{WiseNET SoC}

The WiseNET SoC is the result of a major design effort to reduce power consumption to a minimum $[3,4,5]$. Many RF SoCs have been developed in the last decade mostly supporting mobile phones and connectivity solutions such as Bluetooth or Wi-Fi. These SoCs draw fairly important currents (ranging from a few tens to a few hundreds of $m A$ ) from fairly high $2 \mathrm{~V}$ to $3 \mathrm{~V}$ supplies, in such a way that the battery requires recharging every few hours or days. In applications such as WSN, ultra low-power consumption 
is mandatory in order to sustain applications that can run several years without changing or recharging their batteries. Because many nodes are deployed in a WSN, low cost is also a key issue, which implies using cheap alkaline batteries and therefore designing an RF SoC that can be operated from supplies as low as $1 \mathrm{~V}$ which is the end-of-life voltage for such batteries.

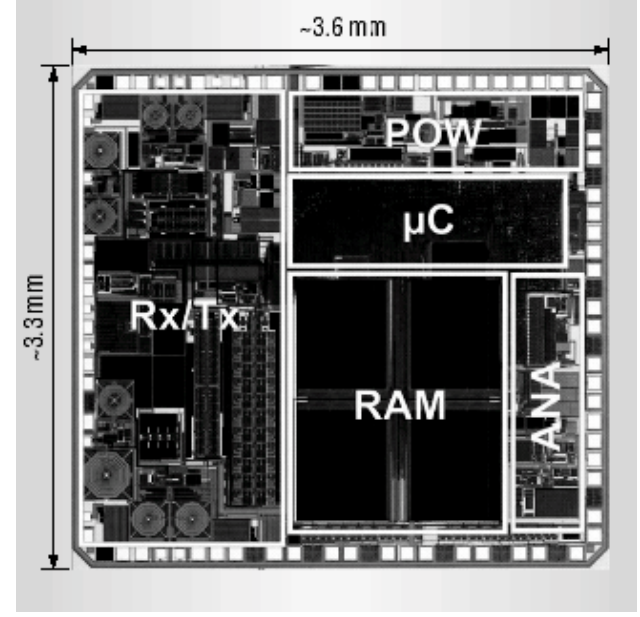

Figure 1: WiseNet chip die picture

The SoC in Figure 1 is integrated in a standard digital 0.18-micron CMOS process from TSMC. Its RF section features an ultra low-power, dual-band $433 \mathrm{MHz} / 868 \mathrm{MHz}$ transceiver for short -range connectivity in the ISM/SRD bands, which operates with $25 \mathrm{~kb} / \mathrm{s}$ frequency shift keying (FSK) or $2 \mathrm{~kb} / \mathrm{s}$ on-off-keyed (OOK) modulations. The SoC embeds also a sensor interface with a signal conditioner, a 10-bit, $10 \mathrm{kHz}$ analog-to-digital converter (ADC); and a 16-bit, sigma-delta, low-frequency ADC. The digital control unit is based on a low-power, 8-bit CoolRISC microcontroller with 22kB low-leakage SRAM. A power management block unit is also included and provides a battery voltage of $1.5 \mathrm{~V}$ to $1 \mathrm{~V}$ to the SoC.

The dual-band operation combined with the use of several channels allows for frequency diversity to solve the difficult problem of rejecting strong nearby interferers. The choice of staying in the $434 \mathrm{MHz}$ and $868 \mathrm{MHz}$ bands instead of moving to the popular and worldwide available $2.4 \mathrm{GHz}$ band is mainly related to power consumption. Indeed, an important part of the power consumption (about $50 \%$ ) of a receiver is directly related to the circuits operating at or close to the RF frequency, such as the frequency synthesizer and the RF frontend (i.e. low-noise amplifier (LNA) and power amplifier (PA)) [3,5]. Since the power consumption of these blocks is approximately proportional to frequency, choosing sub-GHz bands allows to limit the power consumption in the $\mathrm{mW}$ range. Also, it turns out that operating at $2.4-\mathrm{GHz}$ with the chosen $0.18-\mu \mathrm{m}$ CMOS process would require a supply voltage in the order of $1.8-\mathrm{V}$, which is incompatible with the target $0.9-\mathrm{V}$ minimum supply voltage $[3,5]$ unless complex and costly power conversion blocks are used.

For this purpose of achieving lowest possible power consumption, the power requirements must be analyzed for all blocks of the SOC and at all levels:

- Radio Level: The RF SOC achieves $2.5 \mathrm{~mA}$ in receive mode under $1 \mathrm{~V}$ internal supply (hence with a very low $2.5 \mathrm{~mW}$ power budget in active mode). With 1 percent duty cycling, which is affordable for a WSN, it is possible to reach around $25 \mathrm{uA}$ average current consumption, yielding roughly five years of autonomy from a single AA alkaline battery. To achieve this level, a thorough analysis of RF architectures was conducted and led to the selection of a super-heterodyne scheme with a high intermediate frequency. This approach enables the reduction of power consumption in critical highfrequency blocks such as a low-noise amplifier (LNA), first down-conversion mixer and voltagecontrolled oscillator (VCO). In addition, even high-frequency blocks are designed to operate in a weak inversion regime (also known as a sub-threshold regime and generally used for low-frequency analog blocks) whenever possible, clearly demonstrating that RF performance is achievable in 0.18-micron CMOS even with very low-current biasing conditions.

- SOC Level: In addition to the radio, all other parts of the circuit need to yield ultra low-power characteristics. In particular, the SOC is operated by a digital system whose static and dynamic power consumption must be in line with the global $25 \mathrm{uA}$ average target. A major issue is the nonnegligible leakage current for large digital blocks in deep-submicron CMOS, and which is proportional to the area. The digital section occupies a large part of the IC, which is mainly attributed to the $22 \mathrm{kB}$ of on-chip SRAM. To achieve below 3uA of static current (when the SOC is in sleep mode), a 
dedicated SRAM cell is designed and enables an order of magnitude savings on the leakage current thanks to an innovative bulk biasing scheme. The processor's dynamic current consumption, which is directly related to the clock frequency, is also an issue that is addressed with the design of a scalable frequency CoolRISC processor. The latter can be clocked at $6.4 \mathrm{MHz}$ for operations needing speed and as low as $32 \mathrm{kHz}$ for low power. With this approach, power consumption in the digital part of the SOC scales with $50 \mathrm{uA}$ per $\mathrm{MHz}$, which is significantly lower than for solutions using an external offthe-shelf microprocessor.

- CMOS Foundry Level: Designing an SOC in a standard CMOS process is mandatory to reduce wafer costs because no additional process options are used. On the other hand, there is the challenge of designing high-performance RF and analog blocks using baseline MOS and metallization features. For this RF SOC, a dedicated library of RF devices is developed and modeled, and uses only available metal layers for the inductors (no thick metal option), fringe capacitors and MOS device for the varicaps (no mixed-signal or RF options). In addition, clever analog design techniques taking into account the poor characteristics of the baseline CMOS process' MOS devices are used to compensate for process tolerances. With this approach, the RF SOC could be successfully integrated into other foundries without RF and analog performance degradation, and intellectual property (IP) is portable without change.

A die photograph of the WiseNET SoC is given in Figure 1. The IC yields only $3.3 \mathrm{~mm} \times 3.6 \mathrm{~mm}$. A picture of WSN nodes developed at CSEM using the WiseNET SoC is in Figure 2.

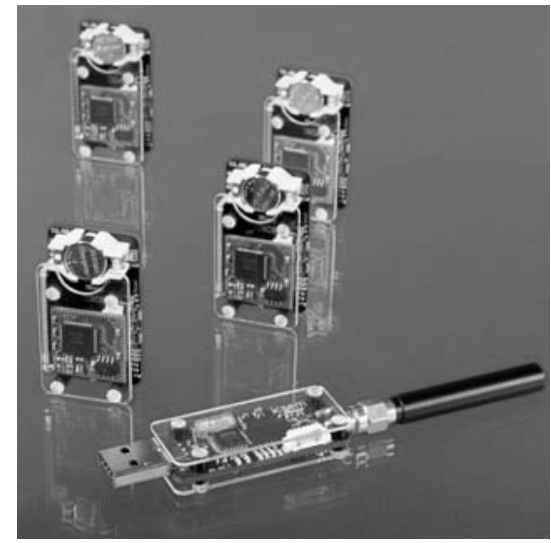

Figure 2: WSN nodes based on the WisenetSOC

\section{WiseMAC}

The low power MAC protocol developed at CSEM for wireless sensor networks is called WiseMAC [2]. It is a contention single channel protocol based on non-persistent carrier sense multiple access (CSMA). Non-persistent CSMA has been combined with reamble sampling to mitigate idle listening. The preamble sampling technique consists in regularly sampling ${ }^{1}$ the medium to check for activity. All nodes in a network sample the medium with the same constant period. Their relative sampling schedule offsets are independent. If the medium is found busy, a node continues to listen until a data packet is received or until the medium becomes idle again. At the transmitter, a wake-up preamble of size equal to the sampling period ${ }^{2}$ is transmitted in front of every data packet to ensure that the receiver will be awake at the start of the data portion of the transmission. This technique permits very low power consumption when the traffic is very low. Using a conventional receiver (i.e. without a second dedicated wake-up radio), it provides, for a given wake-up latency, the lowest possible power consumption in the absence of traffic. The main disadvantage of this basic preamble sampling protocol is that the low power consumption in idle mode is coupled with a high power consumption overhead both in transmission and in reception, due to the wake-up preamble.

In an ad-hoc network, the cost of reception is not only paid by the intended destination, but also by all other nodes overhearing the transmission. The novel scheme introduced by WiseMAC aims at reducing the length of this costly wake-up preamble. This scheme consists in learning the sampling schedule of one direct neighbors, and exploiting this knowledge to use a wake-up preamble of minimized size, as illustrated in Fig. 3. The sampling schedule of a neighbor is learned, or refreshed, during every data exchange by piggy-backing in acknowledgement messages the remaining time until the next sampling

\footnotetext{
${ }^{1}$ In this context, sampling means periodically measuring the received signal strength (RSSI)

${ }^{2}$ In fact, this preamble is created by repeating the packet a number of times up to the desired duration.
} 
time. Every node keeps an up-to-date table of sampling time offsets of all its usual destinations. Since a node will have only a few direct destinations, such a table is manageable even with very limited memory resources.

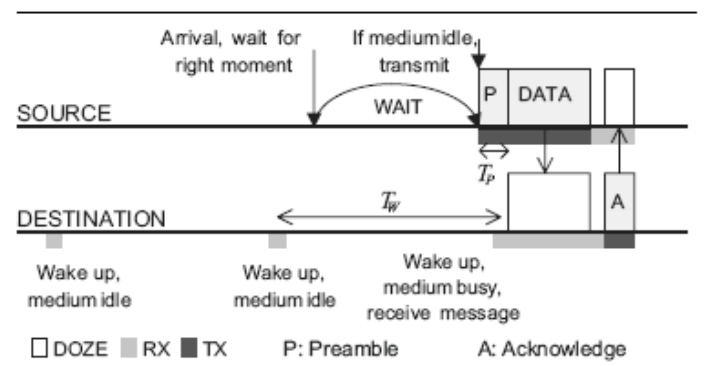

Figure 3: Preamble minimisation with WiseMAC

The duration of the wake-up preamble must cover the potential clock drift between the clock at the source and at the destination. This drift is proportional to the time since the last re-synchronization (i.e. the last time an acknowledgement was received). It can be shown that the required duration of the wake-up preamble is given by $T_{p}=\min \left(4 \theta L, T_{w}\right)$ where $\theta$ is the frequency tolerance of the time-base crystal, $T_{w}$ is the sampling period and $L$ the interval between communications. A transmission is scheduled such that the middle of the wake-up preamble coincides with the expected sampling time of the destination. Systematic collision situations that could have been introduced by this synchronization are mitigated using a wake-up preamble of randomized size.

Because the length of the preamble is proportional to the interval between packets, the overhead of WiseMAC is adaptive to the traffic. WiseMAC can hence both provide an ultra-low average power consumption in low traffic conditions and a high energy efficiency in high traffic conditions. Fig. 4 presents results of simulations in a lattice multi-hop topology. The radio range is chosen such that every node has 8 neighbors. Traffic is flowing through each node with the inter-arrival given on the abscise. It can be seen that WiseMAC can both provide a power consumption below $20 \mu \mathrm{W}$ in low traffic conditions and approach the power consumption of an ideal protocol in higher traffic conditions. With an inter-arrival of 100 seconds, the power consumption amounts to $25 \mu \mathrm{W}$, which translates into more than 5 years lifetime on a single AA alkaline battery. For comparison, Fig. 4 shows also the performance of T-MAC [6] with different duty cycles, of CSMA/CA and of an ideal protocol. All protocols have been simulated using the power consumption and timing parameters of the WiseNET SOC. The power consumption of CSMA/CA is lower bounded by the power consumed in receive mode, as the transceiver is never switched off. The ideal protocol represents the minimum power consumption required to transmit the data without any overhead (idle listening, overhearing, collisions, ...). It is the unreachable bound that low power MAC protocol should try to approach. With the T-MAC protocol, one must select the period of the listen/sleep phases. In the absence of traffic, this ratio is the duty cycle of the transceiver. Every line is stopped as soon as more than $5 \%$ of packets are dropped because of congestion. It can be observed that T-MAC can either provide a low power consumption in low traffic conditions, or a high throughput, while WiseMAC can provide both.

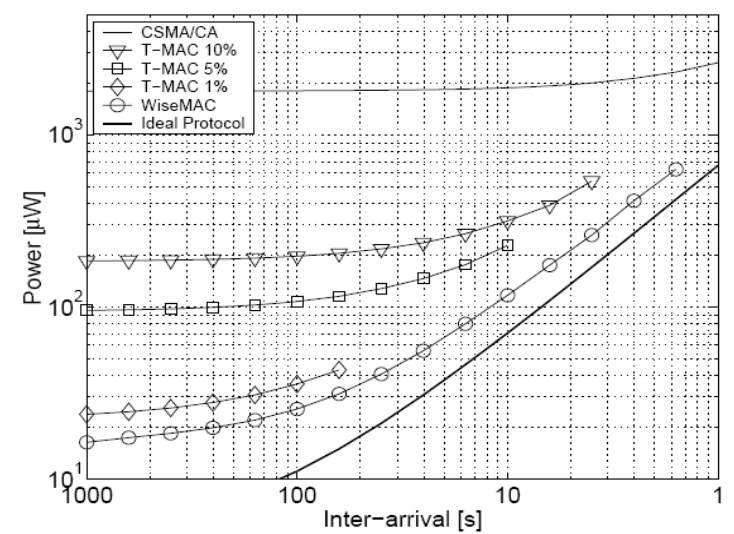

Figure 4: Average power consumption as a function of the traffic 


\section{Higher protocols: routing and code update}

The communication stack is completed by a self configuring cluster-tree routing protocol which is tailored for networks with a limited number of sinks. In such networks, the input data travels from the nodes to the sinks and configuration as well as output data is transmitted from the sinks to the nodes. The tree is created once using limited flooding and the data from the tree structure in unicast from node to node to reach the destination.

The reconfiguration of one or a network of computing devices is the action by which such a system downloads, installs and starts-up a new version of all or part of the program it is running. For instance, the automatic update features (over the Internet) of operating systems running on commonly used personal computers are widely used nowadays. Reconfiguration is not new, but seldom applied in the context of wireless sensor networks, because of the intrinsic constraints: low-footprint, low-power, low data rate, architectural/hardware issues..

The protocol allows the distribution of the new program to every node in the network, originating from the sink, over possibly multiple hops. It is fully reliable and copes with the constraints of WiseMAC. Its basic principle is to use multiple unicast communications to propagate the object in a neighbourhood since the cost of waking-up all nodes is too high, in terms of energy spent. The rules that the individual nodes have to follow to propagate the object are strictly local, ensuring the scalability of the protocol. It also encourages spatial multiplexing by fragmenting the object into pages that progress towards the leaves of the network tree while their follower start to be downloaded near the root.

The patching technique is used to significantly reduce the size of the object to be transmitted over the network. A patch, which represents the difference between the current, running version of the program and the new version to be installed, is sent over the network instead of the full, new program image. The patching technique uses the open-source xdelta 3.0 encoder which computes the difference between two binary files and outputs the result in the VCDIFF format defined in the RFC 3284 . Because the VCDIFF format involved look-up and hash table that are too big for a decoder embedded on a resource constrained node, we introduce a new patch format more adapted to low-resource decoding. The patch in the new format is obtained by translating the VCDIFF patch to the new format. The results presented in the paper show that the new format allows patches which are almost as small as the original VCDIFF patches. Typical compression rates are between 90 and $99 \%$ for small changes and between 65 and $75 \%$ in presence of a major upgrade.

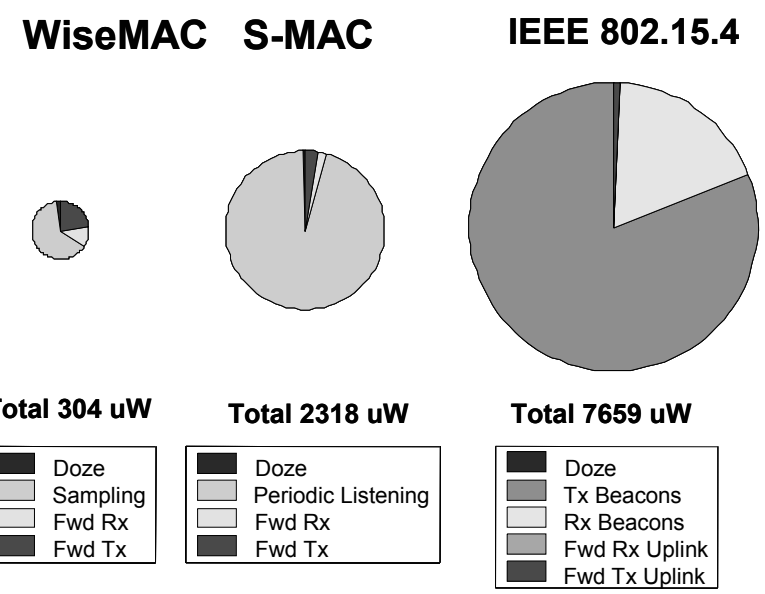

Figure 5: Comparaison of WiseMAC performances using the Semtech XE 1203

\section{Theoretical and Experimental Results}

To assess its performances, WiseNET has first been compared to a few existing solutions. To distinguish between the impact of the SoC and the results of the WiseMAC protocol, the comparison has been done in two steps. In a first step, WiseMAC has been compared theoretically with two other protocols, namely the IEEE 802.15.4 protocol used in Zigbee and the well known S-MAC WSN protocol.

The simulations have been carried out using the radio parameters corresponding to the market reference off-the-shelf XE 1203 transceiver manufactured by Semtech. The average power consumption of a node receiving a 32-byte data packet every 30 seconds and forwarding it to the next node in a multihop network has been calculated. It is assumed that routing is fixed and that no energy is spent in the routing protocol. For S-MAC, we assume a 10\% duty cycle. For IEEE 802.15.4, the node is a ZigBee router and thus that node is coordinator of a beacon enabled PAN and is also associated to its parent PAN. 
The 250-ms wake-up interval is identical for all three protocols. The results are illustrated in Fig. 5. They show that all protocols use little energy to actually receive the message and forward it. However, S-MAC spends most of its energy in idle listening and overhearing, whereas IEEE 802.15.4. exhibits a large overhead due to the combination of the periodic emission of the beacon, the necessity to listen for potential slave emissions and to listen its parent beacon. WiseMAC incurs little overhead because the time necessary to sense the medium is very small. As a result, WiseMAC is about 7 times more efficient than S-MAC and 25 times more efficient than IEEE 802.15.4.

The same simulations have been performed using the parameters of the WiseNET SoC. Because IEEE 802.15.4 spends more time emitting than the other protocols, the consumption ratio degrades. On the other hand, S-MAC that spends most of its overhead listening is favorably impacted by the SoC low receiving energy. WiseMAC is also favorably impacted but to a lesser extent. As a result, WiseMAC uses about 36 times less energy than IEEE 802.15.4 and only a little less than 5 times less energy than SMAC.

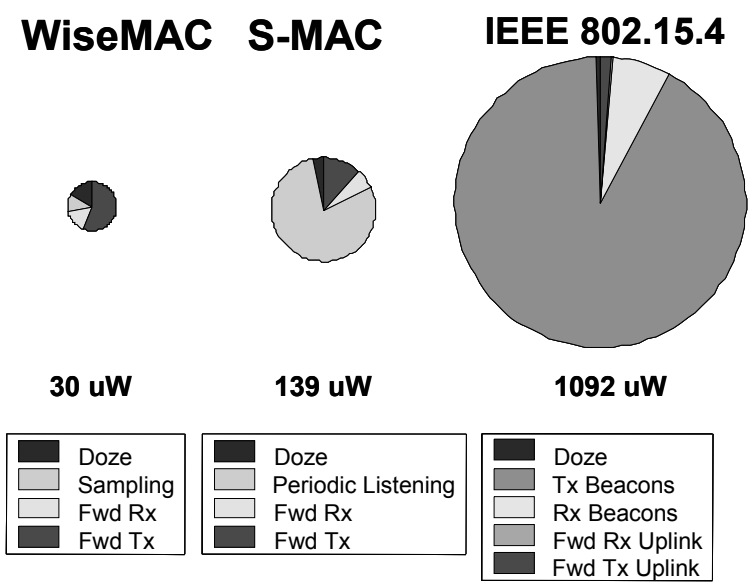

\section{Conclusions}

Figure 6: Comparaison of WiseMAC performances using the WiseNET SOC

Reaching years of autonomy was the objective of the WiseNET project. Impressive energy savings have been reached: 250 times lower than a conventional implementation using off-the-shelf low-power components if we consider IEEE 802.14.5 as a reference, 75 times when compared to S-MAC. This was achieved by a combined optimization of the integrated transceiver hardware and the dedicated medium access control protocol. Additionally, the SoC includes all the basic functions such as data acquisition, processing, storage and communication, that are required by a sensor node and can be powered on low cost alkaline batteries. With an average power consumption of $30-\mu \mathrm{W}$ to forward a 32 byte data packet every 30 seconds with an average of 125 -ms delay per hop, the lifetime is limited by the battery lifetime (around 7 years).

WiseNET provides a complete solution in terms of hardware and protocols including a very low mac, a routing protocol and an efficient code update technique. The hardware and the protocols are best used in combination but may well be used in isolation.

[1] C. C. Enz, A. El-Hoiydi, J.-D. Decotignie, T. Melly, V. Peiris, "WiseNET: An Ultralow-Power Wireless Sensor Network Solution," IEEE Computer Magazine, vol. 37, no. 8, pp. 62- 70, Aug. 2004.

[2] A. El-Hoiydi, J.-D. Decotignie, C. Enz, E. L. Roux,"WiseMAC: An Ultra Low Power MAC Protocol for the WiseNET Wireless Sensor Network," in Proc. 1st ACM Sen-Sys Conf., Nov. 2003, pp. 302-303.

[3] C. C. Enz, N. Scolari, and U. Yodprasit, "Ultra Low-PowerRadio Design for Wireless Sensor Networks," in IEEE Int., Work. on RF Integration Technology, Dec. 2005, pp. 1-17.

[4] V. Peiris, et al., "A $1 \mathrm{~V} 433 / 868 \mathrm{MHz} 25 \mathrm{~kb} / \mathrm{s}-F S K$ 2kb/s-OOKRF Transceiver SoC in Standard Digital 0.18um CMOS," in Int. Solid-State Circ. Conf. Dig. of Tech. Papers, Feb. 2005, pp. 258-259.

[5] C. C. Enz, et al., "WiseNET - An Ultralow-Power Solution for Wireless Sensor Networks," in Analog Circuit Design, J. Huijsing, M. Steyaert, and A. v. Roermund, Eds. Kluwer Academic Publishers, 2004, pp. 91-122.

[6] T. van Dam, K. Langendoen, "An Adaptive Energy-Efficient MAC Protocol for Wireless Sensor Networks", in Proc. 1st ACM SenSys Conf., Nov. 2003, pp. 171-180.

[7] D. Piguet et al., "A remote reconfiguration mechanism for WiseNET wireless sensor networks", First International Workshop on Remote Entrusting, Trento, Oct. 15-16, 2008. 